\title{
A Stochastic Model for Optimizing the Patching Time of Software Bugs
}

\author{
Yong Wang ${ }^{1}$, Dianxiang Xu ${ }^{2}$, William M. Lively ${ }^{1}$, Dick B. Simmons ${ }^{1}$ and Zhaohui Wen ${ }^{3}$ \\ 1. Department of Computer Science, Texas A\&M University, College Station, TX 77843, USA \\ 2. National Center for the Protection of the Financial Infrastructure, Dakota State University, Madison, SD 57042, USA \\ 3. School of Statistics and Applied Mathematics, Anhui University of Finance and Economics, Benbu 233030, P.R. China
}

Received: April 19, 2011 / Accepted: May 19, 2011 / Published: June 25, 2011.

\begin{abstract}
New bugs and vulnerabilities are discovered and reported from time to time even after software products are released. One of the common ways to handle these bugs is to patch the software. In this paper, the authors propose a stochastic model for optimizing the patching time for software bugs and vulnerabilities. The optimal patching time can be computed in the patching script development and operational costs in fix. The authors present two case studies using the Nimda worm vulnerability in Microsoft Internet Information Services web server and the bug report of the Debian project. These studies indicate that the patch applications are later than their optimal fix time.
\end{abstract}

Key words: Optimal patching time, software systems, stochastic renewal model.

\section{Introduction}

Quality assurance of software has been a challenge because modern software is inherently complex. New bugs and vulnerabilities are discovered and reported from time to time even after software products are released. One of the common ways to handle these bugs is to patch the software. There are different types of patches. For example, a patch for proprietary software is often distributed in binary executable. Such a patch modifies existing executable either by including the fixes into the binary file or by completely replacing it. Patches can also be distributed through source code modifications. They consist of textual differences between source codes. In this case, users are expected to compile the new code. Such patches are often observed in open source software systems. In most cases, "patch" means a small and fast fix. Large fixes may use a different name. For example, Microsoft

Corresponding author: Yong Wang, Ph.D., research fields: software engineering, information security, software reliability, software system risk assessment, information security assessment model and metrics. E-mail: johnwangy@gmail.com. calls bulky patches or patches that change a program to a large degree as "service packs" or "software updates". Patches in this paper include all of these types [1].

Bug fixes have their own life cycle, which include such phases as new, open, assign, test, verified, deferred, reopened, duplicate, rejected, and closed [2]. When the bug is posted for the first time, its state is set to "new". After a tester has posted a bug and the bug is approved, the test leader will change the state to "open". After the bug is assigned to a specific developer, the state is changed to "assign". Once the developer develops the script, he/she will send fix code for next round of testing. In this stage, the state of the bug is changed to "test". The bug has been fixed and the patch is released to the test team. "Deferred" means the bug will be fixed in next release. There are several reasons for "Deferred" bugs: The bug has a low priority; there is a lack of time for release, and the bug has no major impact on the software. If the developer feels that the bug is not new, the state of the bug is changed to "rejected". If two bugs are the same, one bug state is changed to "duplicate". If the bug does not appear in 
the software, the bug is fixed, then the state of the bug is changed to be "verified". If the bug still exists after the bug is fixed by developers, the tester changes the state of the bug to "reopened". Once the bug is fixed, it is tested by the tester. The state of the bug is changed to "closed".

Because patches are required to develop and test, there is a time lag between the defect reports and the deployment of patches. When the defects make the software vulnerable, early disclosure of defects or late deployment of security patches can lead hackers to take advantage of vulnerability knowledge. The major cost of patching comes from two major sources: script development and testing and interrupted service when applied to an existing system in operation. Therefore, optimizing patching time for software systems can achieve the best economic benefit when the software systems are in service.

In this paper, we propose a stochastic model for optimizing the patching time for software bugs and vulnerabilities. The optimal patching time can be computed in the patching script development and operational costs in fix. We present two case studies using the Nimda worm vulnerability in Microsoft Internet Information Services web server and the bug report of the Debian project. These studies indicate that the patch applications are later than their optimal fix time.

The rest of this paper is organized as follows: Section 2 presents the model. Section 3 describes two case studies. Section 4 reviewer related work. Section 5 concludes this paper.

\section{Model}

After a bug or vulnerability is reported, it is often desirable to get it fixed. In the following, we discuss the optimal time for fixing it. Let us assume the time of software system failure due to software defects with a distribution function $F(t)$, where $F(t)=P\{T \leq t\} . P\{T \leq$ $t\}$ is the probability of the software system failure within time $t$ due to software defects. The cost to fix the vulnerability or bug by patching is $C_{r}$ [3]. Additional cost will be $C_{f}$ if the software is patched during service and the systems cannot provide the intended service. Let $W_{i}=\left\{T, t_{0}\right\}$ where $\mathrm{t}_{0}$ is the time to fix vulnerabilities and bugs, $\left\{w_{i}\right\}$ is software failure time to provide service and $\left\{w_{i}\right\}$ has identical independent distribution. Let $N_{t}=$ number of bugs or vulnerabilities discovered in $(0, \mathrm{t}]$. If $W_{i}$ has an exponential distribution, $N_{t}$ is the renewal random variable and has a Poisson distribution, thus, $\left\{N_{t} ; t \geq 0\right\}$ is a renewal process.

Let system maintenance cost be associated with the $i$ th week after the software is released with value of $C_{i}$, $C_{i}$ includes the cost from the software patch development (and testing cost) and the cost from the interrupted services for patching purpose. Thus, $\left\{C_{l}\right.$, $\left.C_{2}, \ldots.\right\}$ have an identical independent distribution.

$$
\lim _{n \rightarrow \infty} \frac{1}{t} E\left[\sum_{n=1}^{N_{i}} C_{n}\right]=\frac{E\left[C_{i}\right]}{E\left[W_{i}\right]}
$$

or

$$
\lim _{t \rightarrow \infty} \frac{1}{t} E\left[C_{1}+C_{2}+\ldots+C_{N t}\right]
$$

is an average expected cost incurred in $(0, \mathrm{t}]$.

When we fix time $t_{0}$

Let $Z\left(t_{0}\right)$ denote average cost for $t_{0}$

$$
Z\left(t_{0}\right)=\frac{\text { expected_cost }}{\text { expected_time_length }}=\frac{C_{r}+C_{f} P\left\{T \leq t_{0}\right\}}{E\left[\min \left(T, t_{0}\right)\right]}
$$

where expected_cost is a cost for patching the vulnerabilities or software bugs in software systems, expected_time_length is the expected time for patching the software.

Let $W=\min \left\{T, t_{0}\right\}$

$$
\hat{F}(t)=P\{W \leq t\}
$$

Thus,

$$
\hat{F}(t)=F(t) \text { if } \mathrm{t}<\mathrm{t}_{0} \text { or } \hat{F}(t)=1 \text { if } \mathrm{t} \geq \mathrm{t}_{0}
$$

When $x \geq 0$,

$$
\begin{gathered}
E[X]=\int_{0}^{\infty} x f(x) d_{x}=\int_{0}^{\infty}[1-F(x)] d_{x} \\
E\left[\min \left(T, t_{0}\right)\right]=\int_{0}^{\infty}[1-\hat{F}(t)] d_{t} \\
=\int_{0}^{t_{0}}[1-\hat{F}(t)] d t+\int_{t_{0}}^{\infty}[1-\hat{F}(t)] d_{t}
\end{gathered}
$$




$$
\begin{aligned}
& =\int_{0}^{t_{0}}[1-F(t)] d_{t} \\
& =\int_{0}^{t_{0}} \bar{F}(t) d_{t}
\end{aligned}
$$

Thus,

$$
\begin{gathered}
Z\left(t_{0}\right)=\frac{\text { expected_cost }}{\text { expected_time_length }} \\
=\frac{C_{r}+C_{f} P\left\{T \leq t_{0}\right)}{E\left[\min \left(T, t_{0}\right)\right\}}=\frac{C_{r}+C_{f} F\left(t_{0}\right)}{\int_{0}^{t_{0}}[1-F(t)] d_{t}}
\end{gathered}
$$

To minimize the cost, we need to take $d Z\left(t_{0}\right) / d t_{0}=0$, so,

$$
\begin{gathered}
\frac{d}{d t_{0}} Z\left(t_{0}\right) \\
=\frac{\left[\int_{0}^{t_{0}}\left[1-F(t) C_{f} f\left(t_{0}\right)-\left[C_{r}+c_{f} F\left(t_{0}\right)\right]\left[1-F\left(t_{0}\right)\right]\right]\right.}{\left[\int_{0}^{t_{0}}\left[1-F(t) d_{t}\right]\right]^{2}} \\
\frac{d}{d t_{0}} Z\left(t_{0}\right)=0 \\
{\left[\int_{0}^{t}\left[1-F(t) d_{t}\right] C_{f} \frac{f\left(t_{0}\right)}{1-F\left(t_{0}\right)}=C r+C_{f} F\left(t_{0}\right)\right.}
\end{gathered}
$$

For any $F$, its hazard rate is defined as

$$
\begin{gathered}
h(t)=\frac{f(t)}{1-F(t)} \\
h(t)=\lim _{\Delta t \rightarrow 0} \frac{P\{t<T \leq t+\Delta t\}}{P\{T>t\}} \\
=\lim _{\Delta t \rightarrow 0} P\{t<T<t+\Delta t \mid T>t\}
\end{gathered}
$$

For exponential and Weibull distribution, we can calculate the hazard rate.

For exponential distribution,

$$
\begin{gathered}
h(t)=\frac{1-e^{\lambda \Delta t}}{1-\left(1-e^{-\lambda t}\right)}=\frac{1-e^{\lambda \Delta t}}{e^{-\lambda t}}=e^{\lambda t}-e^{\lambda(t-\Delta t)} \\
h(t)=\frac{f(t)}{1-F(t)}=\frac{\lambda * e^{-\lambda t}}{e^{-\lambda t}}=\lambda
\end{gathered}
$$

It is memoryless. Hazard rate is a constant.

For Weibull distribution, if $\alpha>1$, we have an increasing failure rate (IFR):

$h(t)=\frac{f(t)}{1-F(t)}=\frac{-e^{-(\lambda t)^{\alpha}} *(-\alpha)(\lambda t)^{\alpha-1}}{e^{-(\lambda t)^{\alpha}}}=\alpha \lambda(\lambda t)^{\alpha-1}$

If $\alpha=1$, it is exponential distribution.

From

$$
\frac{d}{d t_{0}} Z\left(t_{0}\right)=0
$$

We can have

$$
\begin{aligned}
& {\left[\int_{0}^{t_{0}}[1-F(t)] d_{t}\right] * h(t)=\frac{C_{r}}{C_{f}}+1} \\
& {\left[\int_{0}^{t_{0}}[1-F(t)] d_{t}\right] * h(t)-F(t)+1=\frac{C_{r}}{C_{f}}+1}
\end{aligned}
$$

The optimal $t_{0}$ for IFR distribution is such that

$$
\left[\int_{0}^{t_{0}}[1-F(t)] d_{t}\right] * h(t)+[1-F(t)]=1+\frac{C_{r}}{C_{f}}
$$

In Eq. (4), $h(t)$ is hazard rate from the software failure either from the software bugs or vulnerabilities. $C_{r}$ is a cost for patching development for the vulnerabilities or bugs. $C_{f}$ is a cost associated with software system failure while the software systems cannot provide the intended services. $F(t)$ is the probability for the system failure in the software systems from the vulnerabilities or bugs.

\section{Case Studies}

In general, patch is applied to software bugs or vulnerabilities. In the following, we will apply the proposed model to both security vulnerabilities and general software bugs. First, we will present a case study on software vulnerabilities. From the software systems in operation until the vulnerability report, we call blind safety period. During this period of time, hackers do not exploit the vulnerability. Our subject system is a web-based application using IIS web server. Nimda is a self-executing virus attack IIS web server and Microsoft outlook [4]. The worm attacks Microsoft outlook users using blank message with an attachment in readme.exe file. According to the websites from the bugtraq in http://www.securityfocus.com and from http://www.secunia.com, the Nimda worm can attack IIS web server 4.0/5.0 version by reading documents outside of the web root. The vulnerability possibly allows executing the arbitrary commands by using malformed URLs that contains Unicode encoded characters. This vulnerability is also called web server folder traversal vulnerability. The vulnerability was reported on October 17, 2000 in CVE-2000-0884. Several variations of Nimda such as Nimda.A, Nimda.C, Nimda.D, Nimda.E were reported from 2001 to 2004. 
The patch for the basic Nimda worm was released on August 15, 2001. The time span from the vulnerability report to the patching fix takes approximately 42 weeks. Patch development and testing are the major initial cost. The past experience for a similar vulnerability patching indicates that the actual probability mass function for security failed time is as follows in Table 1. For simplicity, we assume that security failure always occurs before the end of the week. The patching can be made at the end of week without any interruption to the operation schedule. In this case, patching cost is basic patching development cost of 3,000 dollars. If the security failed before patching, an additional cost of $\$ 1,000$ is incurred because the software systems can't provide intended services. One patch normally can fix one or more reported vulnerabilities. What is the optimal patching time?

According to the model we propose, we can calculate the optimal $z(k)$ value to identify which week is the best patching time in Table 2. From the analysis, we know the week 34 is the best time to patch the software system because average cost $z(k)$ value in week 34 is the lowest based on our proposed model. Therefore, ideally, we need to patch software systems to prevent further economic losses from security breaches earlier than the patch release.

In the following, we present another case study on patching. According to a survey, software bug fix ranges from one hundred days to three hundred days. The medium range of fix days is about two hundreds of days. Let us look at the Debian project patch release and bug fixes. The Debian project is a group of people who contribute a free operating system. The operating system that is created is called Debian GNU/Linux or simply Debian. For details about Debian project, please refer to the project website at http://www.debian.org.

In Debian project, there are bug report databases. The bug reports in Debian project are public available. Although Debian project is an open source project. In reality, the patch development still costs developers time and effort. Therefore, there is a cost related to patch development. We justify that we could use
Table 1 Mass function of security failure distribution.

\begin{tabular}{clll}
\hline Week & Probability & Week & Probability \\
\hline 1 & 0.00 & 22 & 0.01 \\
2 & 0.01 & 23 & 0.02 \\
3 & 0.01 & 24 & 0.01 \\
4 & 0.01 & 25 & 0.01 \\
5 & 0.01 & 26 & 0.00 \\
6 & 0.01 & 27 & 0.02 \\
7 & 0.01 & 28 & 0.01 \\
8 & 0.01 & 29 & 0.02 \\
9 & 0.01 & 30 & 0.02 \\
10 & 0.01 & 31 & 0.02 \\
11 & 0.01 & 32 & 0.04 \\
12 & 0.01 & 33 & 0.08 \\
13 & 0.01 & 34 & 0.06 \\
14 & 0.01 & 35 & 0.06 \\
15 & 0.01 & 36 & 0.10 \\
16 & 0.01 & 37 & 0.07 \\
17 & 0.01 & 38 & 0.10 \\
18 & 0.01 & 39 & 0.08 \\
19 & 0.01 & 40 & 0.04 \\
20 & 0.01 & 41 & 0.025 \\
21 & 0.01 & 42 & 0.05 \\
\hline & & &
\end{tabular}

Debian project bug report as a valid example. Around December 5, 2008, several bugs were reported. These included bug tract number 507913, 507915, 507916 etc. These bugs can cause system crash due to specific inputs. Several months late, there is a patch release to fix the problems on April 9, 2009. Time from the bug reports to the patch release is about eighteen weeks. The probability of operating system failure is listed in Table 3. In the computing, fix scripts are developed in cost of 3,500 dollars, interrupted service for patch will cost 1,000 dollars. The optimal cost for patching is computed. The results are presented in Table 4. From the computation, we know that week 16 is optimal time to patch the Debian operating system since $Z(k)$ value is 410.69 . After week 16 when we patch the Debian operating system, the cost is not optimal.

\section{Related Work}

Arbaaugh et al. (2000) defined a vulnerability life-cycle model in seven stages sequentially [5]. The seven stages are birth, discovery, disclosure, publicity, scripting, correction, and death. Koetzle et al. (2004) 
Table 2 Optimal patching time for the vulnerability in a software system.

\begin{tabular}{lllllll}
\hline Week & $\mathrm{f}_{\mathrm{k}}$ & $\mathrm{F}_{\mathrm{k}}$ & $\mathrm{Cr}+\mathrm{C}_{\mathrm{f}} \mathrm{F}_{\mathrm{k}}$ & $F_{k}$ & $\sum_{i=0}^{k-1} F_{k}$ & $\mathrm{Z}(\mathrm{k})$ \\
\hline 1 & 0.00 & 0.00 & 3,000 & 1.00 & 1.00 & $3,000.0$ \\
2 & 0.01 & 0.01 & 3,010 & 0.99 & 1.99 & $1,512.5$ \\
3 & 0.01 & 0.02 & 3,020 & 0.98 & 2.97 & $1,017.0$ \\
4 & 0.01 & 0.03 & 3,030 & 0.97 & 3.94 & 769.0 \\
5 & 0.01 & 0.04 & 3,040 & 0.96 & 4.90 & 620.4 \\
31 & $\ldots$ & $\ldots$ & $\ldots$ & $\ldots$ & 26.21 & 127.4 \\
32 & 0.02 & 0.34 & 3,340 & 0.66 & 26.83 & 126.0 \\
33 & 0.04 & 0.38 & 3,380 & 0.62 & 27.37 & 126.4 \\
34 & 0.08 & 0.46 & 3,460 & 0.54 & 29.85 & 122.0 \\
35 & 0.06 & 0.52 & 3,250 & 0.48 & 29.59 & 122.3 \\
36 & 0.06 & 0.58 & 3,580 & 0.42 & 29.84 & 124.4 \\
37 & 0.10 & 0.68 & 3,680 & 0.32 & 29.99 & 125.7 \\
38 & 0.07 & 0.75 & 3,750 & 0.25 & 30.12 & 128.4 \\
39 & 0.10 & 0.85 & 3,850 & 0.15 & 30.15 & 130.5 \\
40 & 0.08 & 0.93 & 3,930 & 0.13 & 30.16 & 131.7 \\
41 & 0.04 & 0.97 & 3,970 & 0.03 & 17.29 & 132.5 \\
42 & 0.03 & 0.99 & 3,995 & 0.005 & 0.00 & \\
\hline
\end{tabular}

Table 3 Mass function of system failure distribution.

\begin{tabular}{llll}
\hline Week & Probability & Week & Probability \\
\hline 1 & 0.00 & 10 & 0.04 \\
2 & 0.02 & 11 & 0.05 \\
3 & 0.03 & 12 & 0.06 \\
4 & 0.04 & 13 & 0.07 \\
5 & 0.05 & 14 & 0.08 \\
6 & 0.06 & 15 & 0.08 \\
7 & 0.06 & 16 & 0.08 \\
8 & 0.05 & 17 & 0.10 \\
9 & 0.05 & 18 & 0.08 \\
\hline
\end{tabular}

specified the time period of increased security risk between disclosure and correction stages as time period in "days of risk" [6]. So, we can calculate days of risk by subtracting the date a vulnerability was public reported from the day the vendors released the fix patch. Once a vulnerability is fixed in correction stage, the vulnerability moves to death stage. Generally, for software systems, patching release is a quick fix for the correction stage.

Different defect sources in software bugs and vulnerabilities may take different time spans to fix the problems. Kim and Whitehead studied how long it takes to fix bugs in software systems in two software related projects. They found that bug fix time ranges from 100 days to 300 days. The median bug fix days take 200 days in software systems and PostgreSQL database systems [7].

For software vulnerabilities, different vendors may take different time span to fix. Microsoft spent several months to develop security fix in 2003. For many years, Microsoft has been criticized for taking longer time to issue patches [8]. According to the report in digital bond, US-CERT vulnerabilities take 3-6 months from disclosure to patch fix [9]. Their research showed that disclosure can pressure vendors to issue patches quickly and improve their software quality. The disclosure also can educate computer programmers and network administrators on new vulnerabilities. Therefore, the overall security quality for software systems may be improved. However, all these research only show passive response from software vendors to vulnerability disclosures. Currently once the software vulnerabilities are reported, a fix patch normally is a quick solution to fix the problems. In this paper, we will present an optimal patching time for software security vulnerabilities and bugs. This is a positive 
Table 4 Optimal patching time for the bugs in a software system.

\begin{tabular}{rllllll}
\hline Week & $\mathrm{f}_{\mathrm{k}}$ & $\mathrm{F}_{\mathrm{k}}$ & $\mathrm{Cr}+\mathrm{C}_{\mathrm{f}} \mathrm{F}_{\mathrm{k}}$ & $F_{k}$ & $\sum_{i=0}^{k-1} F_{k}$ & $\mathrm{Z}(\mathrm{k})$ \\
\hline 1 & 0.00 & 0.00 & 3,500 & 1.00 & 1.00 & $3,500.0$ \\
2 & 0.02 & 0.02 & 3,520 & 0.98 & 1.98 & $1,778.1$ \\
3 & 0.03 & 0.05 & 3,550 & 0.95 & 2.93 & $1,211.6$ \\
4 & 0.04 & 0.09 & 3,590 & 0.91 & 3.84 & 934.6 \\
5 & 0.05 & 0.14 & 3,640 & 0.86 & 4.70 & 774.4 \\
6 & 0.06 & 0.20 & 3,700 & 0.80 & 5.50 & 672.7 \\
7 & 0.06 & 0.26 & 3,760 & 0.74 & 6.24 & 602.6 \\
8 & 0.05 & 0.31 & 3,810 & 0.69 & 6.93 & 549.8 \\
9 & 0.05 & 0.36 & 3,860 & 0.64 & 7.57 & 5.17 \\
10 & 0.04 & 0.40 & 3,900 & 0.60 & 8.72 & 477.4 \\
11 & 0.05 & 0.45 & 3,950 & 0.55 & 9.21 & 453.0 \\
12 & 0.06 & 0.51 & 4,010 & 0.49 & 9.63 & 446.3 \\
13 & 0.07 & 0.58 & 4,080 & 0.42 & 9.99 & 423.7 \\
14 & 0.08 & 0.64 & 4,140 & 0.36 & 10.27 & 414.4 \\
15 & 0.08 & 0.72 & 4,220 & 0.28 & 10.47 & 410.9 \\
16 & 0.08 & 0.08 & 4,300 & 0.20 & 10.57 & 410.7 \\
17 & 0.10 & 0.90 & 4,400 & 0.10 & 10.67 & 416.3 \\
18 & 0.10 & 1.00 & 3,500 & 0.00 & & 425.7
\end{tabular}

response for software system development companies to fix the software vulnerabilities and bugs after the software systems are released and the defects are reported.

Stochastic renewal process has been widely used in many applications domains, ranging from manufacturing systems to different computer systems. For example, Rupe and Kuo (1998) applied renewal process to a manufacturing system which consists of multiple machines [10]. Each machine has multiple parts which may have different kinds of failures. They derived a performance model using renewal process. Stochastic renewal process also has been used in computer networks and systems [11]. In a renewal process, inter-arrival times for all arrivals have independently and identically distributed. A renewal process is a general case of a Poisson process. Zhu et al. (2002) assumed that soft real-time tasks arrive in renewal process [12]. These tasks have random service requirements and deadlines in a real-time system. They used queueing theory to computer the long run percentage of tasks which miss the deadline. Belzunce et al. (2006) compared expected failure times for several replacement policies [13]. They compared the expected failure times of a block replacement policy with a renewal process with no planned replacements. In this paper, we plan to apply the renewal process to software systems to estimate optimal software patching policy for software vulnerabilities and bugs after software systems are released.

\section{Conclusions}

In this paper, we developed stochastic model to optimally estimate the fix time of software vulnerabilities and bugs. In the model, we assume that the fixing time of software vulnerabilities and bugs belongs to an exponential distribution and the number of defects discovered belongs to a Poisson distribution. The probability of software security failure is a statistical estimate. From the case studies, we found the date of actual patch release from the software owner is not optimal time for vulnerability fix. There are several reasons for this. One of important reasons may be that software vulnerabilities and bug are in low priority. The other reason may be that software vendors do not realize the potential risk that software system defects may cause. 
Late fix of vulnerabilities may cause software systems to fail in providing reliable services. It could cause a significant cost when the software systems are not available because of security failures. Active planning to fix the software vulnerabilities can make the software systems more reliable in providing intended services. In the similar way, we optimally estimate software bug fix time according to previous failure probability distributions. The proposed model can be applied to any kinds of software defect patching fix.

\section{Acknowledgment}

The authors wish to thank Dr. Richard Feldman at Texas A\&M University for advising stochastic renewal model.

\section{References}

[1] Wikipedia, Patch (computing), available online at: http://en.wikipedia.org/wiki/Patch_(computing).

[2] Bug Life Cycle \& Guidelines, available online at: http://www.exforsys.com/tutorials/testing/bug_life_cycle guidelines.html.

[3] S.M. Ross, Stochastic Processes, 2nd ed., John Wiley \& Sons, Inc., New York, 1996.

[4] K. Pousen, 'Nimda' Worm Hits Net, available online at: http://www.securityfocus.com/news/253, 2001.
[5] W. Arbaugh, W. Fithen, J. Mchugh, Windows of vulnerability: a case study analysis, Computer 33 (2000) 52-59.

[6] L. Koetzle, C. Rustein, N. Lambert, S. Wenninger, Is Linux More Secure than Windows? Forrester Research, available online at: www.forrester.com/Research/Docume nt/Excerpt/0,7211,33941,00.html, 2004.

[7] S.H. Kim, E.J. Whitehead, How long did it take to fix bugs, in: Proceedings of International Workshop on Mining Software Repositories, Shanghai, China, 2006.

[8] M. Franz, US-CERT Vulnerability Disclosure, available online at: http:/www.digitalbond.com/index/php/2006/05 /16/us-cert-livedata-iccp-vulnerability-note/, 2006.

[9] B. Krebs, Security Fix, available online at: http://voices.washingtonpost/securityfix/2006/01/a_time_ to_patch, 2006.

[10] J. Rupe, W. Kuo, Performability of systems based on renewal process models, IEEE Transactions on Systems, Man and Cybernetics-Part A-Systems and Humans 28 (1998) 691-698.

[11] T.G. Robertazzi, Computer Networks and Systems: Queueing Theory and Performance Evaluation, 3rd ed., Springer-Verlag New York, Inc., New York, USA, 2000.

[12] H.F. Zhu, J.P. Hansen, J.P. Lehoczky, R. Rajkumar, Optimal partitioning for quantized EDF scheduling, in: Proceedings of the 23rd IEEE Real-Time Systems Symposium, 2002.

[13] F. Belzunce, E.M. Oretega, J.M. Ruiz, Comparison of expected failure times for several replacement policies, IEEE Transactions on Reliability 55 (2006) 490-495. 